\title{
Contact-line speed and morphology in vertical deposition of diluted colloids
}

\author{
M. Giuliani* and W. González-Viñas ${ }^{\dagger}$ \\ Department of Physics and Applied Mathematics, University of Navarra, Irunlarrea s/n, 31080 Pamplona, Spain
}

(Received 22 October 2008; published 19 March 2009)

\begin{abstract}
We report experimental results on the speed of a receding contact line, in a vertical deposition configuration, during the deposition of polystyrene colloidal particles. We study the effects of the initial concentration of the suspension and compare the measured speeds with the corresponding dried deposit. It is observed that multilayer structures are associated with high speeds. This result is explained through a region in the bulk of the suspension where the concentration of particles increases until multilayer is formed depending on the porosity of the previously deposited structures.
\end{abstract}

DOI: 10.1103/PhysRevE.79.032401

PACS number(s): 83.80.Hj, 47.57.J-, 81.16.Dn

In the last few decades, research directed toward obtaining mesoscopic-ordered materials from colloidal suspensions increased [1-8]. Methods derived from the LangmuirBlodget technique had attracted the attention of the research community because they lead to relatively good structures at a low cost from several points of view. The vertical deposition technique, which belong to this category, is one of the simplest.

The technological interest of this field has its origin in the potential applications of the formed structures as sensors, gratings, special optical coatings, storage devices, or optical elements to be used in optoelectronics and computing $[4,9,10]$. For these applications to be possible it is necessary to obtain specific target structures, that is, without defects or with specific controlled ones. Nevertheless, it is also of great interest to deepen in the physics behind the ordering as a nonequilibrium phase transition.

Typical vertical deposition experiments lead to samples with a macroscopic striped pattern [11-13] corresponding to either different morphologies or number of layers in the deposit. Although stick-slip motion of the contact line has been argued to be the cause for such stripes $[12,14]$, direct measurement of that motion had not been reported until now. Nevertheless, the time evolution of the speed of the contact line has been obtained adjusting a model to experimental thickness profiles [15]. In that work it is suggested that, in multilayer structures, an increase in the number of layers corresponds to a decrease in the rate of formation.

Also, there are some other reports on in situ process in the deposition of colloidal suspensions with similar systems $[16,17]$ but without measuring the speed of the contact line. Studies in dip coating experiments report the morphological characteristics of the stripes as the speed of withdrawal is changed [11].

In this Brief Report, we evaluate the relevance of the speed of the receding contact line in the colloidal structure obtained by the vertical deposition technique. The null speeds were not considered in order to get insight into the relationship between the deposited structure and the speed of the contact line. We present the main statistical parameters

\footnotetext{
*mgiuliani@alumni.unav.es

†wens@fisica.unav.es
}

through the histograms obtained from instantaneous speeds measured directly from position of the contact line versus time. Also, the time evolution of these speeds is investigated and compared with the observed deposit. Finally, we explain the obtained results on the basis of the existence of a zone where the particles accumulate prior to deposit onto the substrate [18] and its relation with the porosity of the deposit.

The experiments were performed using an evaporation cell (Fig. 1) designed in such a way that these allow direct visualization of the movement of the contact line. The used suspension is an aqueous dispersion of electrostatically stabilized polystyrene colloidal spheres $(1.3 \mu \mathrm{m}$ diameter, polydispersity $=0.039$, and surface density charge $\sim 7 \mu \mathrm{C} / \mathrm{cm}^{2}$ ) obtained from Paulke (Fraunhofer-IAP, Germany). The cell has a rectangular base $(17 \times 1 \mathrm{~mm})$, and it is open to the external atmosphere on its upper side. On the 17 $\mathrm{mm}$ sides, two transparent indium tin oxide (ITO) coated glasses are vertically placed. On the small sides $(1 \mathrm{~mm})$, Teflon ${ }^{\circledR}$ walls of the main body of the cell enclose the system.

Prior to the deposition itself, the substrates were cleaned using the Standard Clean-1 (SC1) of Radio Corporation of America (RCA) protocol. This procedure consists of sonication for $15 \mathrm{~min}$ in acetone and a posterior bathing at $67{ }^{\circ} \mathrm{C}$ for $30 \mathrm{~min}$ in a basic piranha solution (deionized water/ hydrogen peroxide/ammonium hydroxide at a 5/1/1 proportion). Between each stage the substrates are intensively

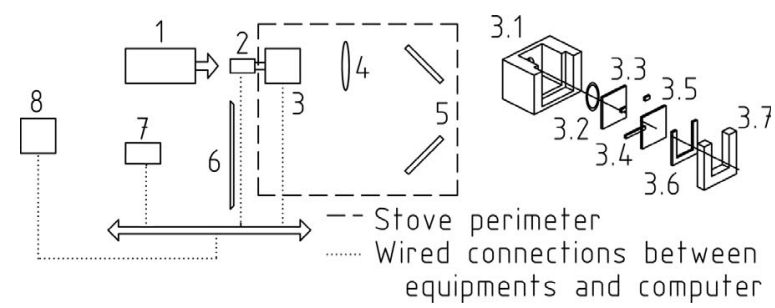

FIG. 1. On the left, a sketch of the experimental system is shown. The dotted lines represent the connections of the different equipment to the computer [(1) cold light source, (2) linear actuator, (3) deposition cell, (4) lens, (5) mirrors, (6) diffuser, (7) video camera, and (8) computer]. On the right, a disassembled sketch of the deposition cell (3) is shown [(3.1) Teflon $®$ body, (3.2) back sealing, (3.3) back substrate, (3.4) ceramic spacers, (3.5) front substrate, (3.6) front sealing, and (3.7) metallic frame]. 


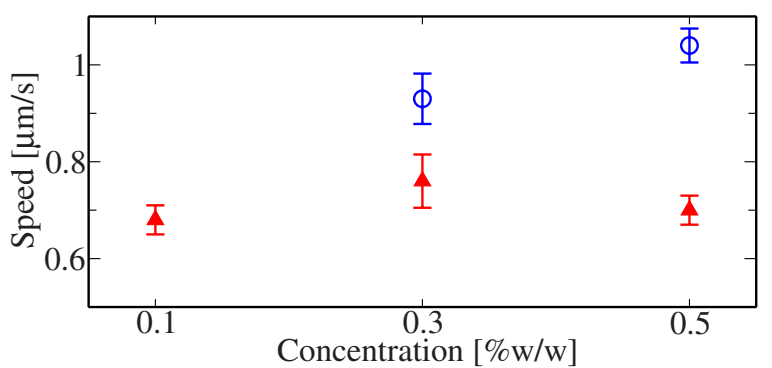

FIG. 2. (Color online) Speed of contact line against initial concentration. The data correspond to the characteristic speed of contact line. Empty circles correspond to an additional speed that appears at more concentrated suspensions.

rinsed with deionized water. Finally, they are dried through $\mathrm{N}_{2}$ blowing. The cell is mounted immediately after this cleaning and placed into an oven at constant temperature $\left(63.0 \pm 0.1^{\circ} \mathrm{C}\right)$. The humidity inside the oven was monitored by means of a thermohygrometer, and it always remained below $2 \%$ relative humidity (RH) and constant during each measurement. Inside the cell, the temperature was measured through two $J$-type thermocouples. Also, the position of the contact line was recorded by a video camera (Fig. 1).

The hydrophilic character of the substrate affects the contact angle and, consequently, the behavior of the contact line. It is known that the cleaning process hydrophilices this kind of substrate $[19,20]$. In addition, this hydrophilicity evolves in time when the substrates are exposed to open atmosphere reaching, asymptotically, an equilibrium state [21]. To obtain identical initial conditions in the substrate hydrophilicity, the suspension was inserted, in all cases, into the cell $22 \mathrm{~h}$ after the cleaning. We further analyze the temporal evolution and main statistical parameters of the speeds. Although the contact angle could play an important role in the mechanism of deposition, the range of concentration used is small and thus also the variation in this angle is (between $60^{\circ}$ and $70^{\circ}$ ). Nevertheless, experiments to evaluate its relevance and effect are being carried out.

First, we consider the characteristic speeds of the contact line. For each initial concentration, we build a histogram of the measured speeds at every position and time. The characteristic speeds are calculated fitting the cumulative histogram with normal distribution functions (usually one or two were enough to obtain a good agreement). The mean value of each distribution is taken to be the characteristic speed and the standard error of the fitting, its error. In Fig. 2, we show the obtained speeds against the initial concentration. It can be seen that for low concentrations $(0.1 \%)$ only one characteristic speed is found. At higher concentrations, one additional peak appears (Fig. 2, empty circles).

Despite the experimental conditions remain constant for each measure, the deposits are not homogeneous. In fact, at micrometer spatial scales, four different morphologies can be found (Fig. 3): multilayer (ML), compact monolayer (CM), noncompact dense (NCD), and noncompact (NC) deposit. The two type of noncompact deposits are distinguished through their clustering properties and particle density. For NC deposit particles form small clusters, mainly, one-

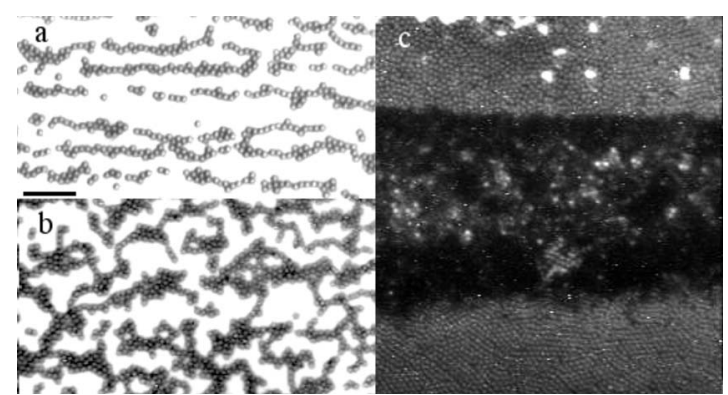

FIG. 3. Micrographies showing typical obtained morphologies: (a) NC, (b) NCD, and (c) ML zone between two CMs. Scale bar is $10 \mu \mathrm{m}$. The contact line has horizontal direction.

dimensional, parallel to the contact line [Fig. 3(a)]. In NCD areas, the deposit consists of bidimensional clusters with some tenths of particles in them [Fig. 3(b)]. The topology of the clusters is related to the shape of the meniscus around the already deposited particles near the contact line [11,22], leading to clusters of different vertical size. The CM structure is characterized for being a monolayer of particles that covers completely the substrate although there could exist small areas (few $\mu \mathrm{m}^{2}$ size) with no particle in it. Every deposit with more than one layer is considered as a ML structure.

For each initial concentration different proportion of these structures are observed. In $0.1 \%$ samples, the deposit is mainly $\mathrm{NC}$, with some thin stripes of $\mathrm{CM}$ ( $\sim 5$-particles-diameter wide). For $0.3 \%$ case, the substrate is covered mostly with NCD structures. In small areas, it is interrupted with ML stripes (vertical or horizontal, Fig. 4) between $\mathrm{CM}$ boundaries. For $0.5 \%, \mathrm{ML}$ and $\mathrm{CM}$ are the most common morphologies. The ML has similar characteristics to the ones that appear at $0.3 \%$.

As it has been shown above, for each initial concentration, the characteristic speeds differ, as well as the predominant morphologies. We also compare the mean value of the strictly positive instantaneous speeds at a given time (Fig. 5, top) with the corresponding dried deposit (Fig. 5, bottom). We observe that, during the periods of high speed, multilayer deposit is being formed (II and IV). When the speed diminishes, the number of layers of the deposit also does (I, III, and V). Performing this comparison with the different deposits we observe that the compact monolayer and the NC deposits are built at similar speeds although the $\mathrm{CM}$ has a slightly smaller one. In the same way, we observe that the NCD structure has a faster associated speed similar to the one measured for the multilayer deposit.

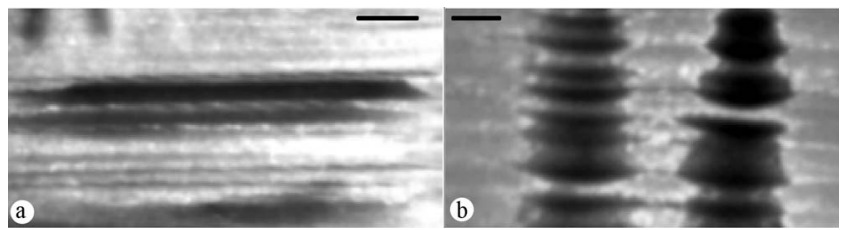

FIG. 4. Low magnification micrographies of the dried deposit obtained for $0.3 \%$ initial concentration. (a) From top to bottom: NCD (bright area) followed by ML (dark) and CM (midtone), finally the deposit returns to NCD. (b) A NCD deposit between two ML vertical stripes. Scale bars are $0.5 \mathrm{~mm}$. 


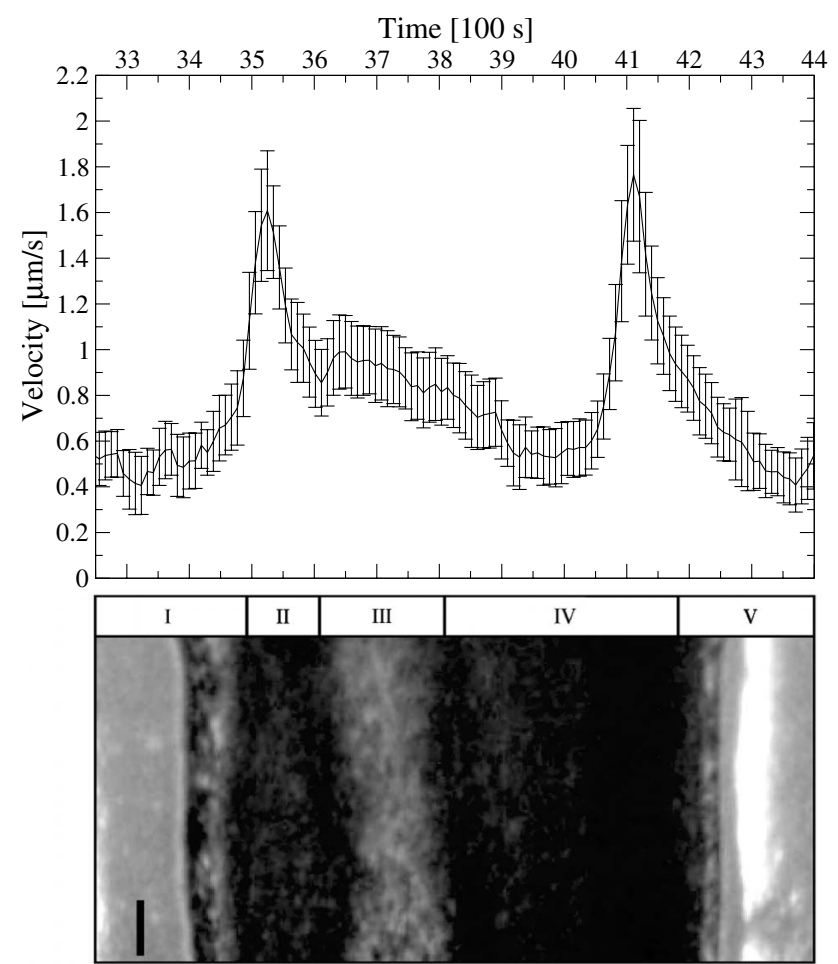

FIG. 5. Top: velocities along the contact line against time. Bottom: deposit micrography. The data correspond to a $0.5 \%$ initial concentration sample. The scale bar is $50 \mu \mathrm{m}$ long. The different regions are the following: I (monolayer), II (multilayer), III (monolayer), IV (multilayer), and V (monolayer and empty space).

The correlation between the temporal evolution of the speeds and the dried deposit allowed us to identify that, in general, $\mathrm{CM}$ and $\mathrm{NC}$ are associated with low speeds, while NCD and ML structures are usually observed at larger speeds.

The most recent models used to explain the formation of this kind of deposit [15] predict that thick deposits need small speed of formation in order to allow enough particles to migrate from the bulk suspension to the area where the structure is been formed. These models had been used in experiments with growing rates much smaller than in our case. In fact, the capillary number in our system is $\mathrm{Ca}$ $\sim 10^{-8}$, about two orders of magnitude higher. Therefore, the different experimental conditions could explain this discrepancy.

In 2006, Shimmin et al. [18] showed that for $1 \mu \mathrm{m}$ polystyrene spheres it was expected that the particles will accumulate in a region near the free surface as the continuous phase is evaporated. From here on, we will name this region the particle pool zone (PPZ). As the concentration in the PPZ is much higher than in the bulk suspension, more particles are available and consequently the multilayer can be built at faster rates. Additionally, the formation of the multilayer reduces the number of particles in this zone thus the deposit should change to one with less number of particles on it (NC, $\mathrm{NCD}$, or CM). Later on, the PPZ zone is rebuilt and the deposit returns to one of the multilayer kind. This alternating process explains the typical striped pattern of deposits obtained by vertical deposition method. Examples of these oscillation can be observed in Figs. 4 and 5.
In Fig. 4 we showed two different multilayer kind of patterns. Figure 4(a) can be explained assuming simultaneous formation of the multilayer over the contact line. When the PPZ is depleted, the structure turns into a monolayer or submonolayer one. In the other situation [Fig. 4(b)], the ML deposit starts to grow in a small area where the meniscus is deformed in such a way that lateral flows appear [23]. These flows drag the PPZ from the neighboring regions toward the area where the ML is being grown. Thus, the ML structure is built below the already deposited one.

The particles are deposited onto the substrate due to the flows that arise from the evaporation of water into the deposited structure. The strength of these flows is increased if the evaporation rate does. Furthermore, at constant temperature and humidity, the evaporation will increase if more surface is exposed, and this will depend upon the height that the water can reach in the structure deposited on the substrate. Thus, it is expected that the strength of the flows differ from one structure to another.

In the noncompact structure, the deposit is very sparse. As a consequence, the meniscus would be very similar to the one without deposit. In compact monolayer structures, the substrate is covered with the polystyrene (PS) hydrophobic spheres, therefore the water is not allowed to go trough the small interstices easily. This results in smaller surface than the one appearing in $\mathrm{NC}$ deposits.

For noncompact dense deposit, the clusters of particles allow the water to go up through the channels. The same situation would occur in the multilayer structure, where it has been observed that the water reaches a higher level than in their nearby structures, despite the hydrophobicity of the spheres.

Based on the discussion above, we observe that the CM and NC deposits have the tendency of being built at slow rates. While the NCD and ML structures allow fast building rates. Nevertheless, in order to these rates to be accomplished it is necessary that there exist particles near the meniscus.

Sometimes it is observed in the time evolution plots (Fig. 5 , first half of region IV) a multilayer structure built at rates comparable to the ones of the compact monolayer (region $\mathrm{V}$ and I), while later on the multilayer present high speeds (second half of region IV). This difference is understood considering the capillarity properties of the deposited structure: in the first part of the region IV the wetted structure is a monolayer (Fig. 5, region III) and, therefore, the flows are weak and the growing rate small regardless a multilayer is starting to form. In the last half of the region IV, the wetted area is the multilayer, which can sustain stronger flows and, thus, higher growing rates. A thick multilayer is grown by these strong flows that deplete the PPZ region, in the absence of particles the deposit turns into a less dense structure.

In conclusion, we report the velocities measured during the formation of a colloidal crystal for different initial concentrations. It was possible to distinguish, at least at our experimental conditions (temperature, substrate properties, and concentration), two characteristic velocities: a small one associated to sparse submonolayer and compact monolayer deposits and a higher one associated to dense submonolayer 
and multilayer structures. These results can be understood by taking into account the capillarity properties of the deposited structure and the existence of a denser region in the bulk near the free surface of the suspension for concentrated enough ones. These two features should be included in a model which could reproduce correctly the vertical deposition in diluted colloidal crystals. This model should be nonlinear and nonlocal in the consideration of the previously deposited structure, leading to a variable wetting film thickness. Also, the model should consider the depletion and replenishment of the PPZ reservoir.
We acknowledge fruitful discussions with A. Yethiraj and with Magneto group at the University of Navarra and the technical support of Y. Olaizola's group. We thank R. Narhe for useful comments and F. M. Hess for assistance with the LINUX GPIB package. W.G.V. also acknowledges discussions with R. Sirera and C. Arcos. This work was partly supported by the Spanish MEC (Grants No. MAT2003-02369, No. FIS2007-66004-C02-01, and No. FIS2008-01126) and by the Departamento de Educación (Gobierno de Navarra). M.G. acknowledges support from the Asociación de Amigos de la Universidad de Navarra.
[1] A. S. Dimitrov and K. Nagayama, Langmuir 12, 1303 (1996).

[2] P. Jiang, J. F. Bertone, K. S. Hwang, and V. L. Colvin, Chem. Mater. 11, 2132 (1999).

[3] H. Nakamura, R\&D Rev Toyota CRDL 39, 33 (2004).

[4] C. López, Adv. Mater. (Weinheim, Ger.) 15, 1679 (2003).

[5] F. Meseguer, Colloids Surf., A 270-271, 1 (2005).

[6] P. Braun, S. Rinne, and F. García-Santamaría, Adv. Mater. (Weinheim, Ger.) 18, 2665 (2006).

[7] M. Yoldi, C. Arcos, B.-R. Paulke, R. Sirera, W. GonzálezViñas, and E. Görnitz, Mater. Sci. Eng., C 28, 1038 (2008).

[8] C. Arcos, K. Kumar, W. González-Viñas, R. Sirera, K. M. Poduska, and A. Yethiraj, Phys. Rev. E 77, 050402(R) (2008).

[9] D. Liu et al., Nano Lett. 6, 2375 (2006).

[10] D. J. Norris and Y. A. Vlasov, Adv. Mater. (Weinheim, Ger.) 13, 371 (2001)

[11] M. Ghosh, F. Fan, and K. Stebe, Langmuir 23, 2180 (2007).

[12] L. K. Teh, N. K. Tan, C. C. Wong, and S. Li, Appl. Phys. A: Mater. Sci. Process. 81, 1399 (2005).

[13] M. A. McLachlan, N. P. Johnson, R. M. de la Rue, and D. W.
McComb, J. Mater. Chem. 14, 144 (2004).

[14] E. Adachi, A. S. Dimitrov, and K. Nagayama, Langmuir 11, 1057 (1995).

[15] G. Lozano and H. Míguez, Langmuir 23, 9933 (2007).

[16] L. Meng, H. Wei, A. Nagel, B. J. Wiley, L. E. Scriven, and D. J. Norris, Nano Lett. 6, 2249 (2006).

[17] M. Ishii, M. Harada, and H. Nakamura, Soft Matter 3, 872 (2007).

[18] R. Shimmin, A. DiMauro, and P. Braun, Langmuir 22, 6507 (2006).

[19] Y. Han, D. Mayer, A. Offenhäusser, and S. Ingebrandt, Thin Solid Films 510, 175 (2006).

[20] Y. Bertholet, Ph.D. thesis, Université Catholique de Louvain, Belgium, 2006.

[21] A. Papra, N. Gadegaard, and N. B. Larsen, Langmuir 17, 1457 (2001).

[22] Y. G. Ko and D. H. Shin, J. Phys. Chem. B 111, 1545 (2007).

[23] C. Fustin, G. Gasser, H. Spiess, and U. Jonas, Langmuir 20, 9114 (2004). 